\section{Investigator-initiated trials are more impartial}

SIR - Your Naturejobs Special Report

“Trial blazers" (Nature 442, 480-481; 2006)

highlights the demand for investigators adept at planning, running and managing clinical trials for the pharmaceutical industry.

The industry's share of total biomedical research increased from nearly a third in 1980 to nearly two-thirds in 2000 . Nevertheless, a report documenting combined data from more than 1,100 studies showed that industry-sponsored clinical trials are "significantly more likely to reach conclusions that were favorable to the sponsor than were non-industry studies", possibly because of publication bias or selection of an inappropriate comparator to the drug being evaluated (J. E. Bekelman, Y. Li and C. P. Gross J. Am. Med. Assoc. 289, 454-465; 2003).

Efforts being made by some academic institutions and peer-reviewed journals to control financial conflicts include the disclosure of financial interests by researchers in academia and as authors in peer-reviewed journals; statistical analyses performed by independent academic researchers; and research protocols and results from clinical trials made fully available in registries accessible to the public.

Given that the trend towards industry's increased share of biomedical research will undoubtedly continue, further evaluation of differential bias between investigator- and industry-initiated contracts for clinical trials within academic medical centres is needed. Of particular note in this respect is the fact that some investigator-initiated trials are funded entirely by industry, whereas others are funded via peer-reviewed grants or foundations.

Within academic institutions, for example, trials funded by industry but initiated by the investigators typically have $10-20 \%$ lower overhead costs. They tend to restrict industry sponsors to ensuring adherence to good clinical-practice guidelines and to checking data for serious adverse events, notation errors and omissions. Hence industry has very limited influence on the conduct of the trial. In contrast, trials that are both initiated and funded by industry within academic institutions have higher overhead costs, usually in the range of $30-40 \%$, paid to the academic institution. Such trials allow greater involvement by the industry funder, for example in writing the protocol, training and assisting investigators, and helping in data collection and analysis.

Academic investigators should be encouraged to lead investigator-initiated clinical trials, thereby limiting industry involvement and reducing any bias that may creep into trial protocols, conduct and results. This should result in better patient care via increased knowledge about the safety and efficacy of the drug therapy under evaluation.

Bradley C. Johnston, Sunita Vohra

Department of Pediatrics, University of Alberta, Edmonton, Alberta T6G 2E1, Canada

\section{Biochar trials aim to enrich soil for smallholders}

SIR - Your recent News Feature "Black is the new green" (Nature 442, 624-626; 2006) accurately summarized the origin, discussions and goals of the Terra Preta Nova group at the recent World Congress of Soil Science in Philadelphia.

However, one might be left with the impression that the biochar initiative is solely directed towards agribusiness applications. From the start, this has certainly not been the case. Indeed, innovative biochar field trials involving a variety of crops are currently being conducted in Amazonia by researchers in Embrapa, the Brazilian agricultural research corporation, and INPA, Brazil's national institute of Amazonian research. These trials are specifically designed for implementation by smallholders, who comprise most of the world's farmers. At the same time, the Brazilian researchers are seeking answers to the questions raised in Philadelphia about the native terra preta of Amazonia, even as US and European researchers are taking the concept in new directions.

William I. Woods ${ }^{\star}$, Newton P. S. Falcão†', Wenceslau G. Teixeira:

*Department of Geography, University of Kansas, Lawrence, Kansas 66045, USA

†INPA/CPCA/Solos e Nutrição de Plantas, Av. André Araujo, Noo 3936, Bairro Petrópolis, CEP. 69011-970, Caixa Postal 478,

Manaus - AM, Brazil

tEmbrapa Amazônia Ocidental,

Rod. AM 010 - Km 29, CEP. 69011-970,

Manaus - AM, Brazil

\section{Biochar and biofuels for a brighter future}

SIR - Your excellent News Feature "Black is the new green" (Nature 442, 624-626; 2006) refers to President Bush's announcement that $\$ 150$ million is to be put into converting cellulosic precursors to ethanol - a very modest amount, considering the plight of petrochemicals. I would like to mention here some progress already being made in producing biofuels and platform chemicals from cellulose and hemicellulose precursors.

Almost quantitative yields of levulinic acid - an excellent platform chemical that gives rise to fuel additives, polymers and plastics, and numerous essential chemicals - are obtained from these precursors in the Biofine process (the work of Stephen Fitzpatrick of Biofine Renewables LLC in Waltham, Massachusetts). A 300-tonne-a-day Biofine plant is nearing final commissioning at Caserta, Italy. Similarly, commercial yields of fermentable levoglucosan from cellulose, and furfural from the pentoses in hemicelluloses, have been obtained in pilot studies using the Convertech process, led by Ken Scott of Scott Convertech in Christchurch, New Zealand.

The biomass to fuel such processes will revolutionize agriculture. It will also give rise to sustainable chemical industries, as oil reserves become depleted and oil prices are pushed up by demand, depletion and political dictates. The biorefinery waste is a char. This has a calorific value similar to that of bituminous coal, and our studies indicate that it has, like the Terra Preta de Indio chars, the potential to be an excellent soil ameliorant. And looking at yet another innovation: the future will be bright if we base it on Carboleum, or 'oil from carbohydrate' - the brainchild of Austin Darragh at the University of Limerick - as we say farewell to petroleum.

\section{H. B. Hayes}

Chemical and Environmental Sciences, University of Limerick, Limerick, Ireland

\section{Science networks spread throughout the Arab world}

SIR - I agree with your Editorial "Science in the Arab world" (Nature 441, 1027; 2006) that the move towards better funding of research and scientific activities in the Arab world is welcome, even though this move remains disproportionate to the wealth of the region.

Although the Arab Science and Technology Foundation is doing good work, it is important not to convey the picture that it is the only organization dealing with research in the area. There is great interest in research among governmental and nongovernmental organizations, in addition to individual efforts. The eastern Mediterranean region of the World Health Organization is very active in this respect. In addition, there is a surge in the number of research networks and online journals, particularly in the areas of medicine and clinical research.

A. Abyad

Health Policy and Regulation, General Authority for Health Services for the Emirate of Abu Dhabi, PO Box 5674, Abu Dhabi, United Arab Emirates

Contributions to Correspondence may be submitted to corres@nature.com. They should be no longer than 500 words, and ideally shorter. 